\title{
RELAÇÃo ENTRE SONO E ATIVIDAdE FÍ́sICA DE NONAGENÁRIOS E CENTENÁRIOS DE PORTO ALEGRE
}

Francielle Bonett Aguirre; PUCRS; franciellebaguirre@gmail.com - Projeto Atenção Multiprofissional ao Longevo (AMPAL) do Instituto de Geriatria e Gerontologia da PUCRS, Porto Alegre, Brasil.

Antonia Angeli Gazola; PUCRS; antonia.gazola@edu.pucrs.br - Escola de Medicina da PUCRS; Projeto Atenção Multiprofissional ao Longevo (AMPAL) do Instituto de Geriatria e Gerontologia da PUCRS, Porto Alegre, Brasil.

3 - Victória Albino Furlanetto Araujo; PUCRS; victoria.albino.araujo@gmail.com - Escola de Medicina da PUCRS; Projeto Atenção Multiprofissional ao Longevo (AMPAL) do Instituto de Geriatria e Gerontologia da PUCRS, Porto Alegre, Brasil.

Ângelo José Gonçalves Bós; PUCRS; angelo.bos@pucrs.br - Escola de Medicina da PUCRS; Projeto Atenção Multiprofissional ao Longevo (AMPAL) do Instituto de Geriatria e Gerontologia da PUCRS, Porto Alegre, Brasil.

\section{RESUMO}

Introdução: A atividade física (AF) é considerada um importante recurso não farmacológico associado à qualidade do sono (QS), particularmente prejudicada na população idosa, mas pouco estudada em nonagenários e centenários, faixa populacional com maior crescimento no Brasil. Portanto, o presente trabalho busca relacionar a QS e a AF em nonagenários e centenários. Métodos: análise secundária dos dados do projeto Atenção Multidisciplinar Domiciliar ao Longevo (AMPAL). A coorte iniciou em 2016 com nonagenários e centenários de Porto Alegre, quando foram questionados sobre a AF e à QS. A análise de dados foi feita por meio do teste qui-quadrado. Resultados: Os participantes eram na sua maioria do mulheres, brancos e viúvos, com ensino fundamental completo (primário), entre 90 e 95 anos e moravam com familiares e 23,7\% realizavam AF. Morar com um cuidador foi visto como fator de estímulo para realização de AF entre nonagenários (40\%, $\mathrm{p}=0,0467)$. Os homens, com $34 \%$, mostrando maior taxa de AF que as mulheres com a frequência de 20\%, ( $p=0,0191)$. Estas (as mulheres) apresentaram com maior frequência pior QS $(p=0,1484)$. Os analfabetos também apresentaram pior QS $(p=0,0100)$. Os participantes com AF apresentaram menor frequência de queixas do sono sendo significativa para a apatia e sonolência diurna $(29 \%, p=0,0170)$. Conclusão: Há escassa literatura acerca da qualidade de sono dessa faixa etária de idosos. Mesmo com as limitações do estudo observamos uma relação importante entre AF e queixas de sono em nonagenários e centenários, observação inédita na literatura.

Palavras-chave: Exercício físico; Distúrbios do início e da manutenção do sono; Idoso de 80 Anos ou mais. 